\title{
Diffusion and adsorption of precursor gas in foam nickel rod substrate during CVD process for deposition of graphene
}

\author{
Bo Tang1 ${ }^{\star}$, Guoxin $\mathrm{Hu}^{2}$ and Dong Huang ${ }^{3}$ \\ 'School of Petroleum Engineering, Changzhou University, No. 88 Xingyuan Road, Changzhou city 213016, the People's \\ Republic of China \\ ${ }^{2}$ School of Mechanical and Power Engineering, Shanghai Jiaotong University, No.800 Dongchuan Road, Shanghai 200240, China \\ ${ }^{3}$ Wuxi entry-exit inspection and quarantine bureau, No. 10 Huangxia road, Wuxi, China
}

\section{Article Info}

*Corresponding author:

Bo Tang

School of Petroleum Engineering

Changzhou University

No. 88 Xingyuan Road

Changzhou city 213016

the People's Republic of China

Tel/Fax: +86 51983295530

Email: tangbo@cczu.edu.cn

Received: November 30, 2016

Accepted: December 27, 2016

Published: December 29, 2016

Citation: Tang B, Hu G, Huang D. Diffusion and adsorption of precursor gas in foam nickel rod substrate during CVD process for deposition of graphene. Madridge J Anal Sci Instrum. 2016; 1(1): 16-20.

doi: $10.18689 /$ mjai-1000104

Copyright: @ $\odot 2016$ The Author(s). This work is licensed under a Creative Commons Attribution 4.0 International License, which permits unrestricted use, distribution, and reproduction in any medium, provided the original work is properly cited.

Published by Madridge Publishers

\begin{abstract}
Preparation of graphene by chemical vapor deposition (CVD) mehtod has attracted increasing attention due to the high quality of the resulting samples. However, the relative research on diffusion and adsorption of precursor gas (the first two steps of graphene growth) on the transition metal surface is still insufficiently. In this study, three-dimensional graphene networks (3DGNs) is prepared by CVD approach with a foam nickel rod (FNR) as the template. The diffusion of $\mathrm{CH}_{4}$ in the FNR is discussed. Then, the adsorption of $\mathrm{CH}_{4}$ on the FNR surface is studied by the expended Langmuir equation, and the influences from $\mathrm{H}_{2}$ on the coverage ratio of $\mathrm{CH}_{4}$ and thickness of the resutling $3 \mathrm{DGN}$ is analyzed. In order to describe the dissolution-segregation process of carbon atoms in the FNR, a parameter named "quasi-diffusivity" is proposed to avoid the tedious calculation. Based on this parameter, the relationship between the scale of 3DGNs and growth time can be simulated, and the relationship b etween the thickness of samples and their growth position can be predicted.
\end{abstract}

Keywords: Chemical Vapor Deposition; Graphene; Expanded Langmuir Equation; Quasidiffusivity.

\section{Introduction}

Since its first isolation in 2004, graphene is regarded as a star material for the dyesensitized solar cells (DSSCs) and supercapacitors fields because of its outstanding electrical and mechanical properties [1]. Recently, graphene with various morphologies including large-scale plane graphene, spherical graphene and three-dimensional graphene networks (3DGNs) have been fabricated on $\mathrm{Cu}$ and $\mathrm{Ni}$ substrates by chemical vapor deposition (CVD) method [2]. Ruoff's group found that the deposition mechanisms of graphene on $\mathrm{Cu}$ and $\mathrm{Ni}$ surfaces are similar [3]. Besides experimental results, some theoretical mechanisms have been revealed. Zhang et al. and Meng et al. calculated the thermodynamics of graphene growth on $\mathrm{Cu}$ and $\mathrm{Ni}$ surface by first-principle calculations $[4,5]$. In previous reports, the attention was focused on the behavior of carbon atoms on the substrate surface. However, the diffusion and adsorption of precursor gas on the substrate surface did not arouse enough attention. In fact, these two processes are closely related to the thickness and quality of the resulting graphene. Studying on these processes is important to optimize the growth parameters and understand the growth mechanism of graphene. 
Recently, our group prepared high-quality 3DGNs by CVD approach with the foam nickel rod (FNR) as a template $[6,7]$, and the 3DGNs based DSSCs and supercapacitors show high photovoltaic performance. Here, we further study the growth mechanism and kinetics of 3DGNs during the CVD process. In this study, the diffusion and adsorption properties of $\mathrm{CH}_{4}$ in the porous FNR is discussed. The influence from $\mathrm{H}_{2}$ on the adsorption of $\mathrm{CH}_{4}$ and thickness of the resulting graphene is studied, as well. In order to simplify the calculation on the kinetics of graphene growth, a parameter named "quasi-diffusivity" is proposed. Based on this parameter, the relationship between scale (thickness) of the 3DGNs and growth time (growth position) can be predicted.

\section{Material and methods}

Detailed growth process of the 3DGNs has described in previous reports $[6,7]$. The heat preservation time are $0 \mathrm{~s}, 150 \mathrm{~s}$, $300 \mathrm{~s}, 500 \mathrm{~s}, 800 \mathrm{~s}$ and $2400 \mathrm{~s}$ for varied samples at $1273 \mathrm{~K}$. An added unit was put into the reaction chamber, and the FNR were placed in the unit during the CVD process (Fig. 1, in the real case, the FNR is put into the unit).

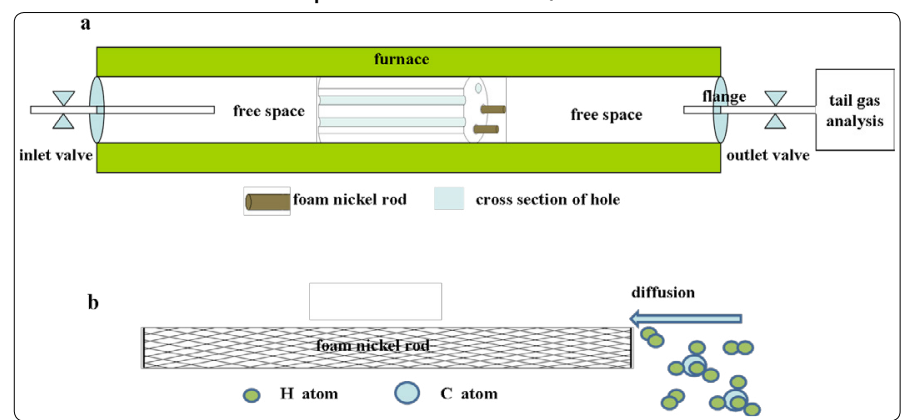

Fig. 1 (a) The schematic of the CVD system. (b) Schematic of the $\mathrm{CH} 4$ and hydrogen molecules diffuse into the foam nickel rod during the heat preservation process.

\section{Results and discussion}

The scale of 3DGNs on the FNR surface increases with the extended heat preservation time (see inset of Fig. 4). No $3 D G N s$ can be found for the sample without heat preservation step, indicating that the growth of the 3DGNs depends on the diffusion of $\mathrm{CH}_{4}$ during the heat preservation process.
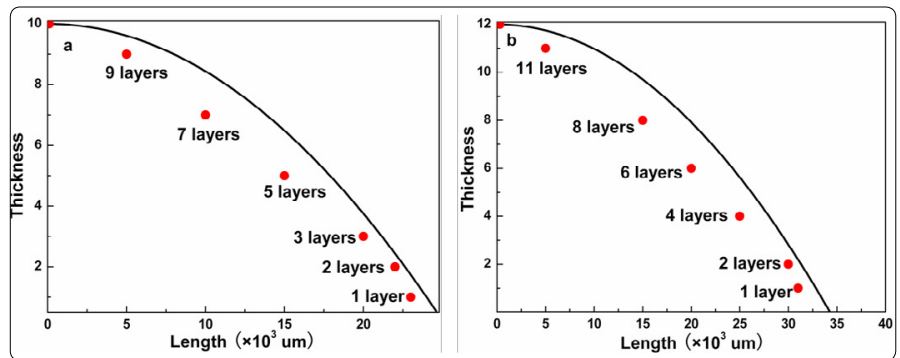

Fig. 4 The simulated and real results of the relationship between the thickness of the 3DGNs and the distance from (a) right and (b) left terminal of the FNR (the sample with 2400s heat preservation time).

\section{Diffusion of methane molecules in the FNR}

The FNR possesses a three-dimensional porous morphology with $100-150 \mu \mathrm{m}$ in porous diameter $[6,7]$. The diffusion law of gas molecules in porous medium is closely related to the relationship between the mean free path of gas molecules and bore diameter of medium. According to the followed formula [8], the values of mean free path of $\mathrm{CH}_{4}, \mathrm{H}_{2}$ and $\mathrm{Ar}$ at $1273 \mathrm{~K}$ are $\sim 380, \sim 660$ and $\sim 470 \mathrm{~nm}$, respectively.

$$
\langle\lambda\rangle=\frac{R T}{N_{A} p \pi d_{0}^{2}}
$$

The $R, T, N_{A^{\prime}} p$ and $d_{0}$ are perfect gas constant, temperature, Avogadro's constant, pressure and gas collision diameter. Due to the mean free paths of these gas molecules (atoms) are far smaller than the pore of the FNR (the ratio less than 0.01), the diffusion of them in the FNR obeys Fick's law. For the sake of simplicity, a uniform pore structure of the FNR is assumed $(\sim 120 \mu \mathrm{m}$ in porous diameter) and the influence from its peripheral boundary is neglected. Therefore, the diffusion of $\mathrm{CH} 4$ molecules in the FNR takes place along the axial direction only and the following one-dimensional partial differential equation can be employed to describe the diffuse process.

$$
\frac{\partial C}{\partial t}=-D^{e f f} \frac{\partial^{2} C}{\partial x^{2}}+u \frac{\partial C}{\partial x}+K C
$$

Where $C, u$ and $k$ are concentration, convection velocity and consumption rate of $\mathrm{CH}_{4}$, respectively. The Deff is the effective diffusivity of $\mathrm{CH}_{4}$ in the $\mathrm{FNR}$, which contains the pore characteristics (poriness $(\varepsilon)$ and tortuosity $(\tau)$ ) of the FNR ( $D^{\text {eff }}=\frac{D \varepsilon}{\tau}, D$ represents diffusion coefficient of $\mathrm{CH}_{4}$ in the free space). In the heat preservation process, the diffusion is an unsteady-state process and the convection velocity equals to zero. According to the results of tail gas analysis, the $\frac{\partial C}{\partial t}$ approximately equals a constant $\left(a \sim 13.6 \mathrm{mgm}^{-3} \mathrm{~s}^{-1}\right)$, thereby, the diffusion equation can be simplified into the following form in the heat preservation process:

$$
D^{e f f} \frac{\partial^{2} C}{\partial x^{2}}-K C=a
$$

The initial and terminal conditions are listed below:

$$
\begin{aligned}
& t=0, \quad x>0, \quad C(x, 0)=0 \\
& t>0, \quad \int_{-L}^{l} C(x, t) d x=Q ; x=l \quad C(x, t)=0
\end{aligned}
$$

Selecting the right part of the FNR as a sample, the calculation can be performed. $L$ is the length of free space in the right side of the unit, and $l$ is the diffusion distance of $\mathrm{CH}_{4}$ in the FNR. $Q$ is the total amount of $\mathrm{CH}_{4}$ in the free space. According to the results of tail analysis, the proportion of $\mathrm{CH}_{4}$ in the reaction chamber is $4.5 \%$ (the calculated value is $4.76 \%$ ) before introducing the unit. After adopting the unit, the proportion of $\mathrm{CH}_{4}$ in the free space (right part) reduces to $4.1 \%$ (the unit separates the chamber into two parts), and the proportion of $\mathrm{CH}_{4}$ in the free space (left part) is $4.7 \%$ by calculation. A visible distinction of the $\mathrm{CH}_{4}$ concentration appears in the right part and left part of the chamber, indicating differences in the scale of the resulting 3DGNs from the right and left parts. If the diffusion of $\mathrm{CH}_{4}$ in these two directions is independent, the diffusivity of $\mathrm{CH}_{4}$ in the right part of the NFR is $\sim 10 \mathrm{~cm}^{2} \mathrm{~s}^{-1}$ at $1273 \mathrm{~K}$. 


\section{Adsorption of methane molecules on the FNR surface}

During the CVD process, $\mathrm{CH}_{4}$ molecules adsorb on the surface of the FNR, which is the previous step of catalytic dehydrogenation reaction. Only the chemisorbed $\mathrm{CH}_{4}$ can make contribution to the growth of $3 \mathrm{DGNs}$, and the dehydrogenation reactions will take place for the chemisorbed $\mathrm{CH}_{4}$ due to the catalytic effect of the substrate. According to Langmuir adsorption model, the covered fraction of adsorbent surface by adsorbate at a given temperature can be expressed as following [9]:

$\theta_{i}=\frac{V_{i}}{V_{\infty}}=\frac{b_{i} P_{i}}{1+\sum_{n} b_{i} P_{i}}$

$\theta, V_{\infty}, \mathrm{b}$ and $\mathrm{P}$ are coverage, maximum capacity of the adsorbent, adsorption coefficient and pressure of the adsorbate, respectively. Subscript i represents varied component, and $n$ is the amount of components.

First of all, the adsorption amount of $\mathrm{CH}_{4}$ molecules on the FNR surface at room temperature (300K) was detected. Six different pressures (101.325, 121.59, 141.855, 162.12, 182.385 and $202.65 \mathrm{KPa}$ ) were adopted, and the corresponding adsorption amounts of $\mathrm{CH}_{4}$ are listed in the Table 1. The equation (5) can be rewritten as the following form:

Table 1 Adsorption amount of $\mathrm{CH}_{4}$ on the FNR surface under varied pressures at $300 \mathrm{~K}$.

\begin{tabular}{ccccccc}
\hline$P(\mathrm{KPa})$ & 101.32 & 121.59 & 141.85 & 162.12 & 182.38 & 202.65 \\
$V \times 10^{-3}\left(\mathrm{~m}^{3} \mathrm{Kg}^{-1}\right)$ & 14.4 & 14.9 & 15.6 & 16.6 & 17.7 & 19.4 \\
\hline
\end{tabular}

$\frac{P}{V}=\frac{1}{\mathbb{B}_{\infty}}+\frac{P}{V_{\infty}}$

The slope and intercept are $\frac{1}{V_{r}}$ and $\frac{1}{W_{W}}$ when the $\frac{P}{V}$ is plotted against the $P$, and the parameters $b$ and $V_{\infty}$ can be calculated according to the curve (Fig. 2). By using identical method, the parameters $b$ and $V_{\infty}$ of $\mathrm{CH}_{4}$ under varied temperatures are calculated (Table 2 ).

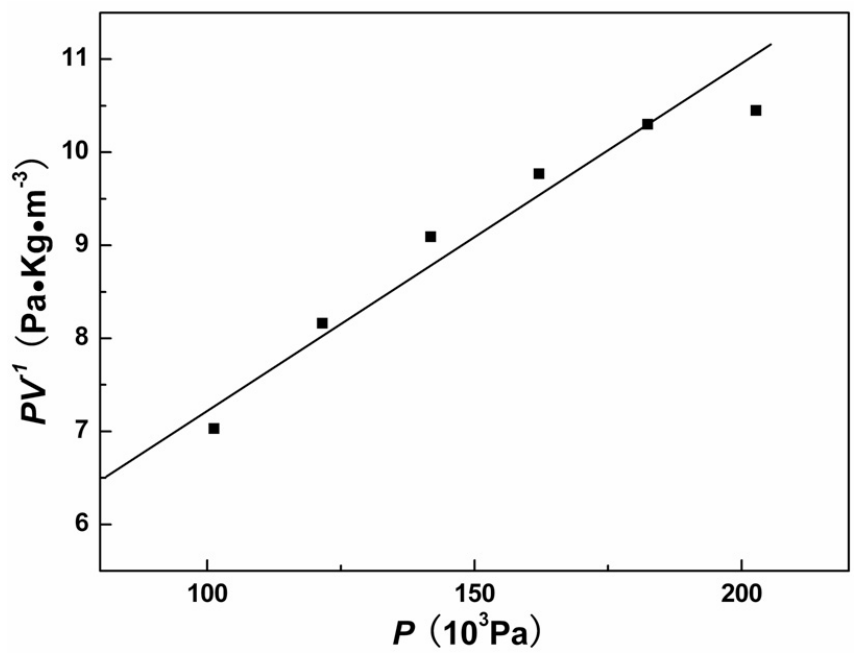

Fig. $2 P V^{-1}$ vs $P$ curves of $\mathrm{CH}_{4}$ adsorption on the FNR.

Table 2 Adsorption parameters of $\mathrm{CH}_{4}$ on the FNR surface under varied temperatures.

\begin{tabular}{ccc}
$V_{\infty} \times 10^{-3}\left(\mathrm{~m}^{3} \mathrm{Kg}^{-1}\right)$ & $28.3(300 \mathrm{~K})$ & $1.1 \times 10^{-2}(1273 \mathrm{~K})$ \\
$b\left(\mathrm{~Pa}^{-1}\right)$ & $1.8 \times 10^{-5}(300 \mathrm{~K})$ & $6.4 \times 10^{-7}(1273 \mathrm{~K})$ \\
\hline
\end{tabular}

Both the $b$ and $V_{\infty}$ decrease with the increased temperature, indicating that high temperature leads to lower coverage of $\mathrm{CH}_{4}$ on the FNR surface. The coverage values of $\mathrm{CH}_{4}$ under varied temperatures are listed in the Table 3. In fact, the calculated values of the $V_{\infty}$ and $\theta$ under high temperature exceed the actual values, and two reasons may lead to this phenomenon. Firstly, the chemical adsorbed $\mathrm{CH}_{4}$ molecules start to decompose on the FNR surface under high temperature, then the resulted carbon atoms diffuse into the FNR and leave surface active sites to adsorb next $\mathrm{CH}_{4}$ molecule. Moreover, nucleation reaction of $\mathrm{CH}_{4}$ would take place in the gas phase under high temperature condition. Therefore, the $V_{\infty}$ value in the Table 2 includes adsorbed and consumed $\mathrm{CH}_{4}$ from above-mentioned two ways. However, the corresponding influence on the $V_{\infty}$ is not significant because the adsorption time is faster than that of nucleation and diffusion process based on composition analysis of the tail gas.

Table 3 Coverage values of $\mathrm{CH}_{4}$ on surface of the substrate under varied temperature.

$\begin{array}{cccc}\theta_{(\%)} & 1273 \mathrm{~K} & 1173 \mathrm{~K} & 1073 \mathrm{~K} \\ & 0.32 & 0.47 & 0.76\end{array}$

In the absence of $\mathrm{H}_{2}$ (the partial pressure of $\mathrm{CH}_{4}$ and $\mathrm{Ar}$ are 5.7 and $95.6 \mathrm{KPa}$, respectively), the calculated coverage of $\mathrm{CH}_{4}$ is $0.36 \%$ at $1273 \mathrm{~K}$. The corresponding coverage of $\mathrm{CH}_{4}$ decreases $\sim 10 \%$ when $\mathrm{H}_{2}$ is added in the atmosphere (the partial pressure of argon, hydrogen and $\mathrm{CH}_{4}$ gases are 57.4, 38.2 and $5.7 \mathrm{KPa}$, respectively), demonstrating that $\mathrm{H}_{2}$ rather than $\mathrm{Ar}$ would depress the adsorption of $\mathrm{CH}_{4}$ on the FNR surface. The adsorption of Ar atoms on the FNR is physical adsorption because Ar gas is an inert gas. Inversely, both $\mathrm{CH}_{4}$ and $\mathrm{H}_{2}$ molecules can be decomposed on the FNR due to the catalysis of transition metals, indicating that both the effective adsorptions of $\mathrm{CH}_{4}$ and $\mathrm{H}_{2}$ are chemical adsorption [0]. Xu et al. and Watwe et al. studied the adsorption heat of $\mathrm{CH}_{4}$ and $\mathrm{H}_{2}$ on Ni surface and calculated the needed dehydrogenation energy of them $[10,11]$. The similar adsorption heat and barrier height for dehydrogenation lead a competition for the surface active sites of the FNR between $\mathrm{CH}_{4}$ and $\mathrm{H}_{2}$. In previous reports, the influence of $\mathrm{H}_{2}$ on graphene growth was proposed [5]: $\mathrm{H}_{2}$ molecules reduce the roughness of $\mathrm{Ni}$ substrates, eliminate impurities (such as $\mathrm{S}$ and $\mathrm{P}$ ) in the $\mathrm{Ni}$ substrates, avoid local variations in the carbon dissolvability and remove defects of graphene at high temperature. Meanwhile, thinner graphene can be prepared on $\mathrm{Ni}$ surface when $\mathrm{H}_{2}$ was introduced in the atmosphere. Koskinen et al. suggested that the dangling bond of carbon atom in the graphene islands would be terminated by hydrogen atom, which limited the thickness of the resulting graphene [12].

Based on the results of this study, the reduced coverage of $\mathrm{CH}_{4}$ shows that the influence from $\mathrm{H}_{2}$ on graphene growth starts from the adsorption stage. The presence of $\mathrm{H}_{2}$ not only terminates the dangling bond of graphene, but also depresses the adsorption of $\mathrm{CH}_{4}$, which reduce the thickness of the asprepared sample. 


\section{Kinetic control step of the 3DGNs}

According to the calculated diffusivity of $\mathrm{CH}_{4}$ in the FNR $\left(\sim 10 \mathrm{~cm}^{2} \mathrm{~s}^{-1}\right), \mathrm{CH}_{4}$ can fill the FNR in several seconds. Therefore, the 3DGNs should cover the whole surface of the FNR, which contradicts to the experimental results. There are two possible reasons can lead to this phenomenon: the $\mathrm{CH}_{4}$ is exhausted or the dehydrogenation reaction only takes place at the endpoint of the FNR. The result demonstrates that $\sim 0.3 \% \mathrm{CH}_{4}$ (the $\mathrm{CH}_{4}$ concentration is $4.1 \%$ before reaction) can be found in the tail gas even the heat preservation time is as long as 2400 s. Therefore, the adsorption and dehydrogenation reactions of $\mathrm{CH}_{4}$ only happen at the endpoint part of the FNR is the fundamental reason. Diffusion velocity of $\mathrm{CH}_{4}$ is slower than the velocity of adsorption and dehydrogenation reactions of $\mathrm{CH}_{4}$ in the surface. The endpoint part of the FNR always possesses enough active sites to chemisorb the $\mathrm{CH}_{4}$, and the resulting carbon atoms diffusion into the FNR both along the axial and radial directions. Thereby, the kinetic control factor of the 3DGNs growth is the diffusion of $\mathrm{CH}_{4}$. The result indicating that the thicknesses of 3DGNs should generally reduce along the axial direction due to the concentration gradient of carbon atoms in the substrate, which is proved by our group $[6,7]$.

\section{Kinetic of 3DGNs growth}

In the cooling process, the decreased solubility of carbon atoms in the FNR leads to the formation of graphene on substrate surface. According to the Langmuir-Mclean model [13], the surface segregation process of carbon atoms complies with the following equation:

$$
\frac{C_{s}}{1-C_{s}}=\frac{C_{\mathrm{b}}}{1-C_{\mathrm{b}}} e^{\frac{-\Delta G_{s e g}}{k T}}
$$

where $C_{\mathrm{s}}$ and $C_{b}$ represent the carbon atoms concentration on the surface and inner of the substrate. The $\Delta G_{\text {seg }}$ is the segregation free energy of carbon atom, $k$ and $T$ represent the Boltzmann constant and temperature, respectively. In fact, the segregation of carbon atoms is a onedimensional diffusion process and the time-dependent equation is:

$$
\frac{\partial C_{b}}{\partial t}=D_{b} \nabla^{2} C_{b}
$$

where $D_{b}$ is the diffusion constant. As for the initial and boundary conditions, the $C_{\mathrm{s}}$ can be assumed as zero at the beginning of the cooling process, and the initial $C_{b}$ can be considered as a constant at specified depth ( $C_{b}(z>0, t=o)=C(x), x$ represent the depth). The bulk concentration of carbon atoms satisfies $\int_{0}^{l} C(x) d x=M$, and the $M$ is the total amount of carbon atoms in the FNR, which equals the change in the weight of the FNR before and after CVD process. However, the $\Delta G_{\text {seg }}$ is a function depending on concentration of carbon atom and temperature, which is difficult to calculate for the varied position of the FNR. Moreover, the precise concentration of carbon atoms at varied position of the FNR before and after cooling process could not to be detected. Thereby, studying the growth kinetic of the 3DGNs through precise calculation is difficult.
In order to simplify the related calculation and give a clear physical picture, we suggest a parameter named "quasidiffusivity" to describe the growth of 3DGNs. In this model, the processes of adsorption and dehydrogenation of $\mathrm{CH}_{4}$ and the processes of diffusion and segregation of carbon atoms are deemed as an equivalent process: the 3DGNs growth in the FNR surface directly. Therefore, the relationship between the scale of 3DGNs and heat preservation time satisfies the following equation:

$$
L=\sqrt{D^{\prime} t}
$$

$D^{\prime}$ is the "quasi-diffusivity", which is not corresponded to any actual process.

With the increased heat preservation time, the scale of 3DGNs increases. The length of the 3DGNs enhances to 2.46 $\mathrm{cm}$ from $0.94 \mathrm{~cm}$ when the heat preservation time increases to 2400 s from 300 s at $1273 \mathrm{~K}$ condition (take right part of the FNR as an example, see inset of Fig. 3a). The calculated $D^{\prime}$ is $0.0028 \mathrm{~cm}^{2} \mathrm{~s}^{-1}$ for the sample with $300 \mathrm{~s}$ heat preservation progress, which is about one-three thousandth of $\mathrm{CH}_{4}$ diffusivity, indicating that graphene can forms on the substrate surface only if the density of carbon atom in the FNR exceeds a certain value. By adopting the $D^{\prime}$, the relationship between heat preservation time and scale of the resulting 3DGNs can be revealed. After comparing the calculated and practical values, it can be found that the fitting values can be used to predict the scale of the 3DGNs. However, the distinctions between the fitting and practical values become obviously when the heat preservation time is expended. Because the total amount of $\mathrm{CH}_{4}$ is unvaried, the amount of carbon atom diffusion into the substrate in unit time gradual reduces. Therefore, the $D^{\prime}$ decreases with the increased heat preservation time. By employing the identical method, the corresponding curve of the samples in the left part is calculated ( $D^{\prime} \sim 0.0041 \mathrm{~cm}^{2} \mathrm{~s}^{-1}$, is calculated by the sample with 300 s heat preservation time, Fig. $3 \mathrm{~b}$ ). The digital image of the whole FNR after 2400s heat preservation time is displayed in the inset of Fig. $3 \mathrm{~b}$. The simulated and experimental results demonstrate that the length of 3DGNs can be designed by controlling heat preservation time, which is important to achieve the controllable growth of the 3DGNs. Moreover, based on the relationship between diffusivity and temperature $\left(D \sim T^{1.51}\right)$, the scale of the 3DGNs under varied temperatures is calculated (Fig. 3).
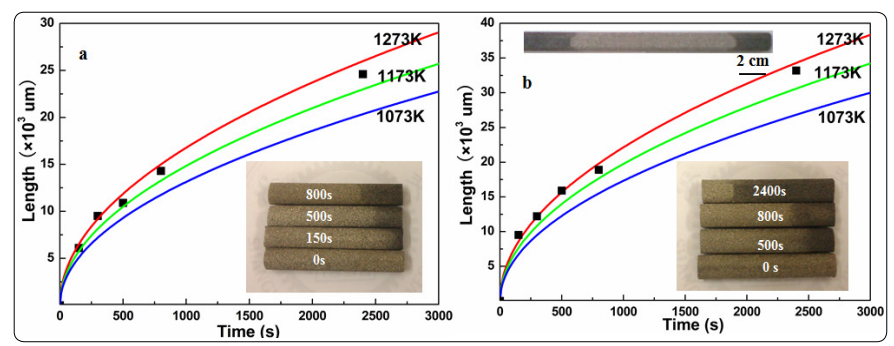

Fig. 3 Experimental and simulation results of the lengths of the 3DGNs from (a) right and (b) left sides of the FNR after varied heat preservation times.

In the cooling processes, due to carbon atoms at varied positions of the substrate possess identical segregation time, 
the different thicknesses of the 3DGNs results from varied densities of carbon atoms at varied positions. Based on "quasidiffusivity", thickness of the 3DGNs at a specific position is proportional to $\sim\left(t_{0}-\frac{d^{2}}{D^{\prime}}\right)$, where $t_{0}$ and $d$ represent heat preservation time and distance from the terminal of the FNR. Therefore, the relationship between thickness of 3DGNs and the growth position can be calculated. The simulated and real results of the 3DGNs in the two sides of the FNR with 2400s heat preservation time (1273K) are displayed in Fig. 4. The results show that the variation tendency of the thickness can be predicted by the curves, although the precision need improving. The "quasi-diffusivity" reduces with time, which results to the gradually increase deviation between the calculated and practical values. In order to obtain the better fitting results, the further optimization for the assumption and approximation is under study.

\section{Conclusions}

The diffusion and adsorption of $\mathrm{CH}_{4}$ on the FNR is studied. The diffusion of $\mathrm{CH}_{4}$ in the FNR complies Fick's law. The coverage of $\mathrm{CH}_{4}$ decrease with increased temperature, and the presence of $\mathrm{H}_{2}$ further reduces the coverage of $\mathrm{CH}_{4}$ due to the competition for the surface active sites. Moreover, the growth kinetic of 3DGNs is studied. The diffusion of $\mathrm{CH}_{4}$ is found the kinetic control factor for the growth of 3DGNs. In order to simplified calculation, "quasi-diffusivity" was proposed to describe the growth process of 3DGNs. By adopting this parameter, the relationship between the scale of 3DGNs and heat preservation time can be obtained. Moreover, a preliminary study on the thickness of the prepared $3 D G N s$ at varied position is performed. All the results manifest that the growth of 3DGNs can be predicted by "quasidiffusivity", which is important to achieve the controllable growth of 3DGNs.

\section{Acknowledge}

This work was supported by National Natural Science Foundation of China under Grant (51506012), Jiangsu Science Foundation Fund under Grant (BK20150266); and the Basic Research Project of Changzhou City under Grant (CJ20159032).

\section{References}

1. Tang B, Hu G.X. Two kinds of graphene-based composites for photoanode applying in dye-sensitized solar cell. J Power Sources. 2012;(220): 95-102. http://dx.doi.org/10.1016/j.jpowsour.2012.07.093

2. Chen ZP, Ren CW, Gao LB, Liu BL, Pei SF, Cheng HM. Three-dimensional flexible and conductive interconnected graphene networks grown by chemical vapour deposition. Nat Mater. 2011;(10):424-428. doi: 10.1038/ nmat3001

3. Li X, Cai W, Colombo L, Ruoff RS. Evolution of graphene growth on $\mathrm{Ni}$ and Cu by carbon isotope labeling. Nano Lett. 2009; 9 (12):4268-4272. doi: $10.1021 / \mathrm{n} \mid 902515 \mathrm{k}$

4. Zhang $\mathrm{WH}, \mathrm{Wu} P$, Li ZY, Yang JL. First-principles thermodynamics of graphene growth on Cu surfaces. J Phys Chem C. 2011;115 (36):1778217787. doi: 10.1021/jp2006827.

5. Meng L, Sun Q, Wang JL, Ding F. Molecular dynamics simulation of chemical vapor deposition graphene growth on $\mathrm{Ni}(111)$ surface. J Phys Chem C. 2012;116(10):6097-6102. doi: 10.1021/jp212149c

6. Tang B, Hu GX Gaob H, Shic Z. Three-dimensional graphene network assisted high performance dye sensitized solar cells. J Power Sources. 2013; (234): 60-68. http://dx.doi.org/10.1016/j.jpowsour.2013.01.130

7. Tang B, Hu GX. Preparation of few layers three-dimensional graphene networks by CVD for energy storage application. Chem.Vapor Depos. 2014 ;20 (1-2-3):14-22. doi: 10.1002/cvde.201207052.

8. $\mathrm{Wu} \mathrm{SH}, \mathrm{Wu} \mathrm{CY}$. Time-resolved spatial distribution of scattered radiative energy in a two-dimensional cylindrical medium with a large mean free path for scattering. Int J Heat Mass Tran. 2001;(44):2611-2619.

9. Ignatowicz K. A mass transfer model for the adsorption of pesticide on coconut shell based activated carbon. Int. J Heat Mass Tran. 2011;54 (2324): 4931-4938. http://dx.doi.org/10.1016/j.jheatmasstransfer.2011.07.005

10. Christmann K, Schober O, Ertl G., Neumann M. Adsorption of hydrogen on nickel single crystal surfaces. J Chem Phys. 1974; 60 (11): 4528-4540. doi: http://dx.doi.org/10.1063/1.1680935

11. Watwe RM, Bengaard HS, Rostrup JR, Dumesic JA, Norskov JK, Theoretical studies of stability and reactivity of $\mathrm{CH}_{\mathrm{X}}$ species on $\mathrm{Ni}$ (111). Journal of Catalysis. 2000;189 (1): 16-30.

12. Koskinen $P$, Malola S, Hakkinen H. Self-Passivating Edge Reconstructions of Graphene. Phys. Rev. Lett. 2008;101(11-12):115502-115505. doi: https://doi.org/10.1103/PhysRevLett.101.115502

13. Mccarty KF, Feibelman PJ, Loginova E, Bartelt NC. Kinetics and thermodynamics of carbon segregation and graphene growth on $\mathrm{Ru}$ (0001). Carbon. 2009; 47 (7)1806-1813. doi: 10.1016/j.carbon.2009.03.004 